Review

\title{
Adjuvant Radiochemotherapy versus Chemotherapy Alone for Gastric Cancer: Implications for Target Definition
}

\author{
Jing $\mathrm{Xu}^{1}$, Jonathan $\mathrm{Zhu}^{2}$, Qichun Wei ${ }^{1 凶}$ \\ 1. Department of Radiation Oncology, the Second Affiliated Hospital and Cancer Institute (National Ministry of Education Key Laboratory of Cancer \\ Prevention and Intervention), Zhejiang University School of Medicine, Hangzhou 310009, P.R. China \\ 2. Ben May Department for Cancer Research, University of Chicago, Chicago, IL, 60637, USA
}

$\square$ Corresponding author: Dr. Qichun Wei, Department of Radiation Oncology, the Second Affiliated Hospital and Cancer Institute (National Ministry of Education Key Laboratory of Cancer Prevention and Intervention), Zhejiang University School of Medicine, Hangzhou 310009, P.R. China. Postal address: Jiefang Road 88, Hangzhou 310009, PR China. Tel: +86-571-87783522; Fax: +86-571-87214404; E-mail: qichun_wei@zju.edu.cn

(C) Ivyspring International Publisher. This is an open access article distributed under the terms of the Creative Commons Attribution (CC BY-NC) license (https://creativecommons.org/licenses/by-nc/4.0/). See http://ivyspring.com/terms for full terms and conditions.

Received: 2018.05.18; Accepted: 2018.10.28; Published: 2019.01.01

\begin{abstract}
The INT0116 trial was a milestone study and laid the foundation for the adjuvant radiotherapy (RT) associated to concurrent chemotherapy (CT) for the treatment of gastric cancer (GC) after gastrectomy. However, it is still controversial whether adding RT to CT could further benefit D2-dissected GC patients. The ARTIST trial indicated that the addition of RT to CT did not have a positive impact on disease-free survival (DFS). Nevertheless, in a subgroup of 396 patients with positive pathological lymph nodes, combined treatment with RT was superior to CT alone. A similar randomized Chinese trial confirmed the superiority of adding RT to CT in terms of DFS for patients with D2 lymphadenectomy. However, several previous randomized studies provided inconsistent results with the benefits of combined treatment of RT and CT. The inconsistent results of several studies may be due to the differences between tumor epidemiology, treatment policies, and treatment outcomes. During the past decade, major progress in accurate target delineation utilizing RT technology has been observed. However, even though the use of adjuvant RT doubled after the INT-0116 trial results became public, the fraction of patients receiving adjuvant RT was still low according to the SEER database. The low rate of adjuvant RT can partially be explained by concern over toxicity while undergoing RT. Several studies have also defined the specific location of locoregional recurrence for postoperative RT in GC, but these studies are still limited. A number of retrospective studies demonstrated that the most prevalent nodal recurrence was outside the D2 dissection field. In order to overcome the restricted nature of a retrospective study and provide more individual radiation field determination, additional large-scale prospective multicenter studies are required to evaluate the optimal RT target.
\end{abstract}

Key words: gastric cancer; radiotherapy; chemoradiotherapy; target definition

\section{Introduction}

Currently, gastric cancer (GC) is one of the most common malignant neoplasms in the world, particularly in the Asia-Pacific region [1]. Even with the use of aggressive treatment, the 5-year survival rate for patients with lymph node positive disease is as low as $15-20 \%$, and even node-negative patients have a 5-year survival rate of only $45-55 \%$ if they reach an advanced $\mathrm{T}$ stage $\left(\mathrm{T}_{3}-\mathrm{T}_{4} \mathrm{~N}_{0}\right)$ [2]. Surgical resection is the mainstay therapeutic modality of non-metastatic GC. The initial results of the randomized control trials revealed similar long term survival from a D1 vs. D2 lymph node dissection [3-5]. However, the benefit of a gastrectomy with a D2 dissection was confirmed in the Dutch trials in terms 
of both locoregional recurrence and GC-related death after a 15-year follow-up [6]. Since there is no difference of local recurrence rate between D2 and D3 surgery, D2 lymph node dissection appears to correspond to a "plateau level" [7]. Gastrectomy with a D2 lymph node dissection is now considered a standard of care and supported by the European Society for Medical Oncology in Europe [8].

The largest series in the literature demonstrated recurrence rates ranging from $20 \%$ to $40 \%$ within 2 years after complete resections performed for curative purposes of GC $[9,10]$. Resected GC recurs in multiple patterns: locoregional, peritoneal, and distant sites are common modes of recurrence [11]. The risk of recurrence depends on specific clinicopathologic factors such as initial stage of the disease, Lauren's histological type, proximal location et al [9]. Since the results of treatment remain dismal, strategies for adjuvant treatment in an attempt to reduce recurrence are warranted. In the current clinical practice, the role of adjuvant chemotherapy (CT) has been established and postoperative $\mathrm{CT}$ is routinely used for D2-dissected GC. To date, the value of adding postoperative radiochemotherapy to $\mathrm{CT}$ is largely unknown, and there is no evidence of clear benefit of the use of radiotherapy (RT) in the post-operative setting [12]. In this review, we discuss the state and the rationale of adjuvant $\mathrm{RT}$ in light of recent evidence and new perspectives to the treatment of GC.

\section{Role of adjuvant $R T$ /postoperative $R T$ in the randomized trials}

The integration of adjuvant RT into the routine treatment of GC has been initiated based on the INT0116 study [13]. In this landmark phase III study, a total of 556 patients with resected GC or cancer within the gastroesophageal junction were randomized to receive surgery alone or surgery plus postoperative RT (45 Gy of radiation at 1.8 Gy per day) associated with 5-fluorouracil based CT. Three-year overall survival rates were $50 \%$ in the chemoradiotherapy (CRT) group vs. $41 \%$ in the surgery-only group $(P=0.005)$. The three-year relapse-free survival rate was $48 \%$ in the CRT group vs. $31 \%$ in the surgery-only group $(P<0.001)$. This benefit from postoperative CRT was confirmed later in an update, published by Smalley et al [14] in 2012, which demonstrated strong persistent benefit from adjuvant CRT. However, this trial has been criticized because lymph node dissection was limited (36\% had a D1 and 54\% had a D0 dissection) and many patients experienced high rates of toxic side-effects. The incidence of grade 3 or higher hematological and gastrointestinal toxicities which existed in $54 \%$ and $33 \%$ of patients, respectively, has indicated the need for improving the treatment safety. High rates of severe toxicity may be explained by the use of the conventional two-dimensional (2-D) RT technique associated with the 5-fluorouracil-based CT regimen. Recently, a retrospective cohort study from a Latin American Center also suggested that there was a long-term overall and disease specific survival benefit for patients treated with postoperative adjuvant treatment for stage IIIB and IIIC GC after D2 lymph node dissection [15]. Similarly, a large retrospective study suggested that the postoperative CRT in D2-resected GC patients could prolong survival and decrease recurrence [16].

The above studies evaluated postoperative CRT versus surgery alone, however, the role of postoperative RT in the treatment of GC was not well established. The most relevant study about the role of postoperative RT following the INT0116 study was the phase 3 Adjuvant chemoRadiation Therapy in Stomach cancer (ARTIST) trial, which investigated the role of postoperative CRT (capecitabine + cisplatin followed by capecitabine and concurrent RT at a dose of $45 \mathrm{~Gy}$ ) vs. CT alone (capecitabine + cisplatin) in 458 patients who underwent D2 lymphadenectomy. Overall, the addition of RT to the CT did not significantly prolong disease-free survival (DFS) $(P=$ 0.0862 ). However, in the subgroup of patients positive for pathologic lymph nodes at the time of surgery $(n=396)$, combined treatment was superior to CT alone $(P=0.0365)$, and the statistical significance was retained at multivariate analysis $(P=0.0471)$. This result supported the hypothesis of the benefit of RT in the adjuvant treatment of patients with lymph node positive disease [17, 18]. A recent clinical trial confirmed that adjusted recurrence-free survival was significantly higher in the CRT group than the CT group in patients with $\mathrm{D} 2$ resected lymph node metastatic GC [19].

In addition to the ARTIST trial, several randomized trials and meta-analyses have tried to explore the comparison of CRT to adjuvant CT in GC patients who undergo D2 lymphadenectomy, but the results were inconsistent [20-28]. Characteristics of the included trials comparing postoperative CRT and postoperative CT were listed in table 1.

In 2012, a Chinese randomized trial compared 45 Gy intensity modulated radiation therapy (IMRT) plus concurrent CT with CT alone in 380 locally advanced GC patients after D2 resection. The median duration of recurrence-free survival in patients receiving CRT was also better $(P=0.029)$, but there was no impact on median overall survival $(P=0.122)$. Multivariate analyses showed that lymph nodes metastases and TNM stage were both independent prognostic factors. There was no difference between 
two treatment groups with respect to rates of all grade adverse events [20]. The National Cancer Center in South Korea conducted a phase III trial that enrolled 90 locally advanced GC patients (stage III/IV M0) who underwent D2 lymph node dissection to compare adjuvant CT alone versus CRT (INT-0116 scheme). Addition of RT to CT could significantly improve locoregional recurrence-free survival (LRRFS) and DFS in stage III GC compared to CT alone. RT in addition to CT has shown a similar toxicity profile as CT alone [21]. Similarly, it was indicated that comparing to CT alone, CRT can increase the one-, two-, and three-year total survival rates, as well as the DFS rates of GC patients (T3/T4 and/or $\mathrm{N}+$ ) who have been initially treated with surgery [22].

Table 1. Characteristics of the included trials comparing postoperative chemoradiotherapy and postoperative chemotherapy.

\begin{tabular}{|c|c|c|c|c|}
\hline $\begin{array}{l}\text { First } \\
\text { author }\end{array}$ & Country & $\begin{array}{l}\text { Year } \\
\text { published }\end{array}$ & $\begin{array}{l}\text { Total } \\
\text { accrual, } \mathrm{n}\end{array}$ & Clinical target volume delineation \\
\hline Lee [18] & Korea & 2012 & 458 & $\begin{array}{l}\text { tumor bed, anastomosis site, } \\
\text { duodenal stump, regional lymph } \\
\text { node, and } 2 \mathrm{~cm} \text { beyond the proximal } \\
\text { and distal margins of resection }\end{array}$ \\
\hline Zhu ${ }^{[20]}$ & China & 2012 & 351 & ICRU 50 report \\
\hline Kim ${ }^{[21]}$ & Korea & 2012 & 90 & $\begin{array}{l}\text { anastomosis site, duodenal stump, } \\
\text { regional lymph nodes, and more than } \\
2 \mathrm{~cm} \text { from the proximal and distal } \\
\text { margins of resection }\end{array}$ \\
\hline $\mathrm{Yu}{ }^{[22]}$ & China & 2012 & 68 & $\begin{array}{l}\text { tumor bed, stroma, and regional } \\
\text { lymph node }\end{array}$ \\
\hline Kwon [26] & Korea & 2010 & 61 & $\begin{array}{l}\text { tumor bed, anastomosis, stump, and } \\
\text { regional lymph node areas }\end{array}$ \\
\hline $\begin{array}{l}\text { Bamias } \\
{[27]}\end{array}$ & Greece & 2010 & 143 & $\begin{array}{l}\text { tumor bed, anastomosis, the stomach } \\
\text { remnant, and regional lymph nodes }\end{array}$ \\
\hline Kilic [28] & Turkey & 2013 & 71 & $\begin{array}{l}\text { tumor bed, anastomosis, and regional } \\
\text { lymph nodes }\end{array}$ \\
\hline
\end{tabular}

A total of 895 patients from 3 randomized controlled trials were identified for a meta-analysis evaluating the comparison of adjuvant CRT vs. solely $\mathrm{CT}$ in patients with GC who underwent D2 lymphadenectomy. An increase in LRRFS $(P=0.01)$ and DFS $(P=0.25)$ in favor of combined treatment was demonstrated, however, there was no benefit in terms of the distant metastasis recurrence-free survival and overall survival. The main grade 3 or 4 toxicities were equivalent between the two groups [23]. In another meta-analysis of 1171 patients, similar results showed that CRT was associated with a significant increase in DFS when compared to CT alone (odds ratio 1.48, 95\% CI 1.08-2.03). However, there was no significant difference in overall survival (odds ratio 1.27, 95\% CI: 0.95-1.71). Five trials found no statistically significant differences in the toxicities between the two groups [24]. Zhou and colleagues reported a meta-analysis of 4 randomized controlled trials comparing CRT with CT for GC after R0 surgical resection. It was suggested that postoperative CRT, compared with postoperative
$\mathrm{CT}$, can improve loco-regional recurrence rate and DFS, but did not improve distant metastasis rate and overall survival [25].

By contrast, there have only 3 notable, randomized trials in the past which have demonstrated the addition of RT to CT did not appear to provide any additional advantage in radically resected advanced GC patients. A total of 61 patients with stage IIIA, IIIB and IV (without metastasis) GC was treated with postoperative CRT. Arm A consisted of one cycle of 5-fluorouracil/cisplatin (FP) followed by $45 \mathrm{~Gy}$ to radiation field with capecitabine. One month after completion of $\mathrm{RT}$, patients received three additional cycles of FP every 3 weeks. Arm B consisted of six cycles of FP. There was no significant difference between the two arms in 5-year DFS (76.7 vs. $59.1 \%$, respectively; $P=0.222$ ) or overall survival (70.1 vs. $70.0 \%$, respectively; $P=0.814$ ) [26]. Despite the results of this study, the sample size was too small to draw any definitive conclusions. In another randomized phase III study, a cohort of 147 radically resected GC patients with stage $\geq \mathrm{T} 3$ and/or $\mathrm{N}+$ were randomized to 6 cycles of docetaxel with cisplatin, both at $75 \mathrm{mg} / \mathrm{m}^{2}$ every 3 weeks (arm A) or the same treatment with RT (arm B; 45 Gy). Due to excessive nausea and vomiting, cisplatin was substituted by carboplatin at AUC (area under the curve) of 5 after the first 45 patients ( 22 group A, 23 group B). It was indicated that the addition of RT to adjuvant CT does not appear to improve survival in high-risk, radically resected GC [27]. A similar study from Turkey also denied the superiority of adding RT to CT in terms of DFS or overall survival for patients who underwent $\mathrm{D} 2$ dissection and were at $\mathrm{pT}_{\text {any }} \mathrm{N}_{3} \mathrm{M}_{0}$ stage [28]. The Lancet Oncology recently published the results of the CRITICS trial evaluating the role of adjuvant RT for western patients with perioperative CT and D2 dissection. The CRITICS phase III multicenter trial is an open-label randomized trial which aims to determine the clinical outcomes in patients undergoing preoperative epirubicin, cisplatin or oxaliplatin, and capecitabine CT followed by surgery with adequate lymph node dissection and concurrent CRT (45 Gy, cisplatin and capecitabine). Postoperatively, 233 (59\%) of 393 patients started CT and $245(62 \%)$ of 395 started CRT. The results of this study demonstrated that postoperative CRT did not improve clinical outcome compared with postoperative CT in patients with resectable GC treated with adequate preoperative CT [29].

Theoretically, application of RT in conjunction with CT may be a better method in the management of GC, because RT might improve locoregional control while systemic CT might diminish or eliminate microscopic distant metastasis. 
Nevertheless, in actual practice, it was still disputed whether adding RT to CT could further benefit those GC patients after adequate lymph node dissection. There were several reasons related to the dissonance of adjuvant RT versus solely CT. First only a limited number of studies, mainly in East Asia, compared the benefit of adding adjuvant RT to the CT alone. Not only were these studies limited, but these studies also contained small sample sizes. The lack of research and participants hinder any conclusions to be made. Second, the RT techniques applied in the trials were different, including conventional AP-PA fields, three-dimensional conformal radiation therapy (3D-CRT) or IMRT. Additionally, CT regimens differed. Compared with the commonly used SOX (oxaliplatin plus S-1) or XELOX (oxaliplatin plus capectabine) regimens, the cytotoxic drugs used in the previously referenced trials may have relatively low efficacy with high toxicities. Meanwhile, considering the poor postoperative patient compliance in the CRITICS trial, future research and trials should focus on optimizing preoperative treatment strategies [30]. Third, the number of lymph nodes dissected differed. For example, in the ARTIST trial, approximately $60 \%$ of the patients with stage IB and II disease had greater numbers of lymph nodes dissected (Reaching up to 40 lymph nodes) which might dramatically lower the risk of locoregional recurrence [31]. Lastly, the inclusion criteria for patients also differed. The subgroup of high-risk patients that present particular characteristics such as positive surgical margin, perineural invasion, and diffuse histological subtype, might benefit more from local irradiation after surgery [12].

\section{Ongoing trials concerning adjuvant RT}

Overall, the outdated RT techniques and CT regimens involved in the clinical trials mentioned above, and the poor trial design might lead to the contradictory results. The ongoing trials concerning the role the adjuvant RT may better define the role of postoperative RT. The ARTIST II trial is currently underway and aims to clarify the benefit of postoperative CRT in patients exclusively with lymph node metastasis. This trial is currently comparing surgery + adjuvant S-1 CT, vs. surgery + adjuvant SOX (S-1 and oxaliplatin) CT, vs. surgery + adjuvant SOX (S-1 and oxaliplatin) CT + RT. The primary endpoint of the study is DFS. The three-arm trial may better define the role of postoperative RT in GC treated with D2 lymphadenectomy as it focuses on patients with stages -II or -III disease with positive lymph nodes.

\section{Incomplete adoption of adjuvant RT for resected GC}

As mentioned, On the basis of the compelling evidence provided by the INT-0116 trial, adjuvant CRT was adopted widely for the treatment of patients after radical surgery. Between 1996 and 2003, 10,230 patients aged 18-85 years with resected GC were identified in the Surveillance, Epidemiology, and End Results (SEER) database and classified as diagnosed before or after presentation of the INT-0116 trial findings. It was demonstrated that the percentage of adjuvant RT use was $14.6 \%$ before the INT-0116 trial, increasing to $30.4 \%$ afterward $(P<0.001)$. Though the use of adjuvant RT doubled after the INT-0116 trial results became public, the fraction of patients receiving adjuvant $\mathrm{RT}$ was still low. In this study, $\geq$ $85 \%$ of the cohort treated after INT-0116 could be eligible for adjuvant RT because they had Stage Ib-IVM0 disease, however, only 30.4\% received adjuvant RT [32]. Similarly, it was reported that only 26 patients $(2.0 \%)$ received adjuvant RT after curative resection in a multiple-center cohort of Chinese patients [33]. If adjuvant CRT usage on eligible patients increased, it would help identify an unambiguous interpretation of the benefits by providing a larger, more concrete sample size with better data of recurrence and locoregional survival rates.

\section{Recurrence patterns after curative surgery for $\mathrm{GC}$}

Concern about toxicity could partially explain the low rate of adjuvant RT in this analysis. In postoperative RT for GC, the definition of clinical target volume (CTV) of the aforementioned clinical trials was listed in table 1 . The target volume range for RT was extremely diverse for the adjuvant RT. Furthermore, a larger margin should be added to the CTV for the respiratory motion and inter-fractional variability, thus increasing the planning tumor volume (PTV) [34]. This leads to large radiation volume for upper abdomen with RT. In light of the INT0116 results using conventional 2-D techniques that $17 \%$ of patients were not able to complete the entire treatment course because of severe acute toxicity [13].

Over the past decade, postoperative RT procedures for GC have shifted from simple 2-D techniques to 3D-CRT or IMRT. In particular, IMRT had the advantage of conformal delivery of high doses to the target area, while decreasing damage to the adjacent normal tissue [35]. It was suggested that IMRT was better than 3D-CRT at protecting the kidneys from postoperative adjuvant radiation, and 
late nephrotoxicity was less severe with IMRT. Hence, IMRT was recommended for the subset of patients with risk factors for kidney disease or those with preexisting nephropathy $[35,36]$. However, it is reported that post-operative GC patients treated with 3D-CRT or IMRT are still at a considerable risk of dose-limiting toxicity to various surrounding organs, such as the liver, spleen, pancreas, et al. [34, 37, 38]. In addition, RT-induced nausea and vomiting are common and troublesome symptoms among GC patients receiving RT. Severe nausea and vomiting may interfere with a patient's performance status and quality of life causing treatment delay or refusal that may compromise tumor control [39]. To overcome these problems, radiation-related toxicity may be reduced by improving RT treatment planning. Furthermore, recurrence patterns (locoregional, peritoneal, or distant/systemic) after curative resection and specific location of locoregional recurrence should be illustrated in detail to generate more accurate radiation volume, which may minimize radiation-related toxic effects.

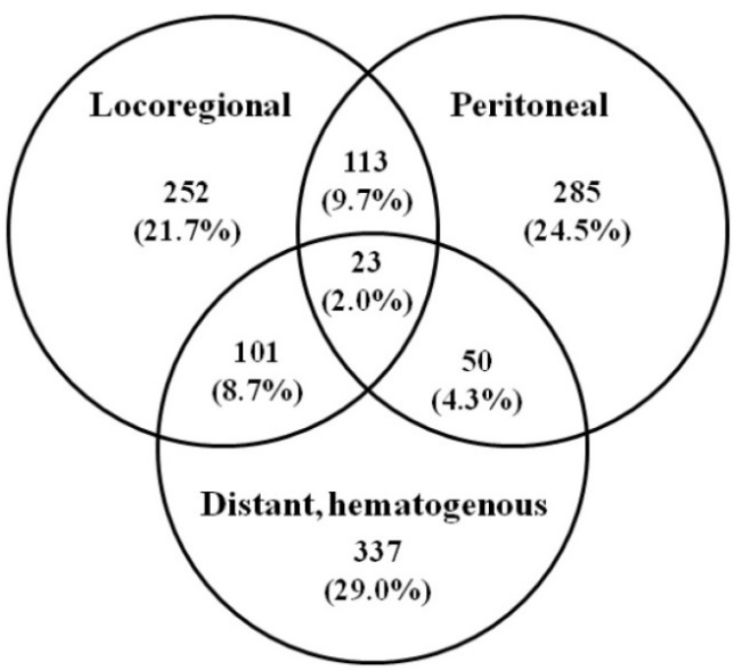

Figure 1. Initial recurrence patterns of 1161 patients after gastrectomy for gastric cancer in 4 literatures. Values in parentheses are percentages.

Recurrence significantly reduces the patient's survival and is usually associated with pain, bleeding, and other symptoms, with no single method of treatment which can efficiently address all variants of GC spread. Most patients succumbed within one year of receiving a diagnosis of recurrence with a mean survival of 8.7 (range 2-66) months [10]. Several studies have attempted to analyze recurrence patterns in GC with variable results. Figure 1 summarized the initial recurrence patterns of 1161 patients after gastrectomy for GC in 4 literatures [9, 10, 40,41]. Of the 1161 patients, 874 patients $(75.2 \%)$ had recurrence involving a single area, $264(22.7 \%)$ had recurrence involving 2 areas, and $23(2.0 \%)$ had recurrence involving all 3 areas. Overall, the locoregional area, distant sites, and peritoneal recurrence involved as any part of the recurrence pattern in 489,511 , and 471 patients $(42.1 \%, 44.0 \%, 40.5 \%)$, respectively. It appears that prediction of recurrence patterns of resected GC based on clinicopathologic factors is elusive [9]. The variability of the data on the recurrence patterns is multifactorial and probably attributable to the selection in patient cohorts, the time at which recurrence was detected, and the methods for determining recurrence patterns. As RT is a locoregional treatment, improved survival is likely explained by better locoregional tumor control. It was recommended that three sites must be included in the adjuvant RT after radical surgery, including the primary tumor bed, the anastomosis or duodenal stumps, and the regional lymphatics [42].

\section{Remnant stomach}

Whether remnant stomach should be included in the RT target still remains controversial. One of the major variations in radiation fields was the remnant stomach, which was included in the INT-0116 trial but not in the ARTIST trial [43]. It was suggested that if necessary, the remnant stomach in patients who underwent a subtotal gastrectomy (STG) has been included [44].

However, Chang et al. suggested that only 15 of 1952 patients who underwent STG had a local relapse in the remnant stomach. It therefore seemed safe to exclude the remnant stomach from the potential field of RT [45]. Similarly, Nam et al. retrospectively analyzed 291 GC patients treated with STG and D2 dissection and adjuvant CRT. Eighty-three patients underwent irradiation according to the INT 0116 protocol, in which the target volume routinely included the remnant stomach (Group A). Remnant stomach excluded from the target volume for 208 patients (Group B). There was no significant difference for overall and DFS rates between the two groups of patients with and without remnant stomach irradiation. Hence, exclusion of remnant stomach from the radiation field had no effect on failure rates or survival, and it seemed reasonable not to consider the remnant stomach as a target volume for adjuvant RT [43].

\section{Tumor bed, anastomosis or stumps}

Most clinical series have reported similar results, with the local recurrences comprising $13 \%-22 \%$ of all recurrences after D2 gastrectomy [46]. Though the incidence of local recurrence was suggested to be the lowest among the recurrence patterns, the site most prone to local recurrence was the anastomosis or stump (15.7\%) [10]. Especially the patients with distal 
antral tumors that extend beyond the gastric wall toward the retroperitoneal structures or pancreas are suggested to have increasing incidence of positive margins. Given the high risk of tumor bed-anastomotic/stump relapse, it is imperative to include the tumor bed and longitudinal surgical margins in adjuvant RT treatment design. The location of the anastomoses/stump must be ascertained in all patients [42].

\section{Lymph node considerations}

Understanding the location of regional lymph node recurrence have raised increasing alarm allowing individualized RT strategies and rational tailoring of RT target identification. Location of local regional failure after gastric resection defines the postoperative RT fields needed when adjuvant RT is employed. Careful delineation of regional failure patterns is imperative to proper conceptualization of RT treatment planning. Gastric lymph node involvement is closely associated with the site of the primary tumor, making its site indispensable for a proper definition of gastric portions.

However, until now, only a few retrospective studies had defined the specific location of locoregional recurrence for postoperative RT in GC. In a Korea study, the most common site of locoregional recurrence was regional lymph nodes $(23.8 \%)$ after curative gastrectomy and D2 lymph node dissection in patients with stage III (N3) GC. The most prevalent nodal recurrence was in lymph node stations No.9 (lymph nodes along the celiac artery), No.12 (lymph nodes in the hepatoduodenal ligament), No.13 (lymph nodes on the posterior surface of the pancreatic head), No.14 (lymph nodes along the superior mesenteric artery or superior mesenteric vein), and No.16 (lymph nodes around the abdominal aorta), which were outside the D2 dissection area [47]. The results were similar to findings from Chang et al, demonstrating that the locoregional relapse at 5 years after gastrectomy with D2 lymphadenectomy was 8.5 percent, and the most commonly involved site was station No.16b1 (paraaortic lymph nodes between the lower border of the left renal vein and the upper border of the origin of the inferior mesenteric artery) [45]. The cause might be a lymphatic flow which ultimately drained into the station 16 , the paraaortic region, regardless of the location of the primary tumor [45]. Similarly, after D2 dissection in stage III GC with N3 disease, the most commonly involved first recurrent lymph nodes were suggested to be No.16b (lymph nodes around the abdominal aorta from the upper margin of the left renal vein to the aortic bifurcation, 61.5\%) and No.16a (lymph nodes around the abdominal aorta from the upper margin of the celiac trunk to the lower margin of the left renal vein), regardless of the primary tumor location. Besides no. 16, commonly involved recurrent lymph nodes were the Nos.9, 10 (lymph nodes in the splenic hilar), and 13 nodes in the proximal third of the stomach $(30 \%$, $10 \%$, and $10 \%$, respectively); Nos.12 and 14 nodes in the middle third (26\% and $13 \%$, respectively); Nos. 12 , 13, 14, 9, and 11 (lymph nodes along the splenic artery) nodes in the distal third (39\%, 27\%, 20\%, 20\%, and $10 \%$, respectively); and Nos. 14, 12, 11, 9, 2 (lymph nodes on the left pericardial) if the primary tumor was located in more than two-thirds of the stomach $(41 \%, 24 \%, 12 \%, 12 \%$, and $12 \%$, respectively) [44]. If lymph node metastases exceeding $10 \%$ is regarded as a high risk, these lymph node stations are recommended to be included in the CTV delineation of regional lymph node.

$\mathrm{Yu}$ et al. analyzed the results of the ARTIST trial, and found that the regional area (LNs in groups 2 and 3 including the paraaortic, retropancreatic, aortocaval, retrocaval region) might be the most important RT target [48]. This can be explained by the fact that initial drainage is usually to lymph nodes along the lesser and greater curvatures which are removed with most types of gastrectomy. Therefore, optimizing regional control in stations 13-16 is important.

In an attempt to better define which locoregional lymph nodes are at risk, Smalley et al. [42] reviewed the literature and concluded that the involvement of the splenic hilar nodes in lower-third/antral tumors was sufficiently low $(<10 \%)$ to allow consideration for exclusion in this clinical setting but that subpyloric pancreaticoduodenal nodes have a much greater incidence (nearly 50\%) [42]. In contrast, tumors involving the upper one-third of the stomach have a low incidence of subpyloric lymph node metastasis $(<10 \%)$, but were more likely to involve the splenic nodes (12-42\%) [42]. Regardless of any generalizations, these findings offered the important information in modifying radiation fields from "standard" and supplied the opportunity for individualized treatment planning according to the location of the primary tumor within the stomach.

The Japanese data showed that the relative risk of nodal involvement at a specific nodal site was dependent on the primary GC localization [49]. In addition, there are several clinicopathological aspects relating with the lymphatic drainage regions and affecting radiation target definition, including histologic differentiation, tumor size, macroscopic type, and depth of tumor invasion [50]. For male GC patients with large, deeply invasive, poorly differentiated, diffusely infiltration and positive cancer embolus, the radiation fields were suggested to be enlarged appropriately [51]. Overall, for radiation 
oncologists, all these related clinical and pathological parameters should be taken into account for defining appropriate individualized field arrangements for the adjuvant postoperative RT of GC.

A large inter- and intraobserver variability in target volume delineation was reported in GC patients [52, 53]. In a questionnaire, $75 \%$ of respondents indicated the elective nodal area as the major subjective difficulty [54]. Nodal stage is based on the number of involved LNs in the AJCC staging system for GC, which does not reflect the rules of lymphatic drainage and provides no practical indications for delineating the target volume. Accordingly, in the future, international consensus guidelines are needed to be thorough and clear about the detailed indications for the irradiation of each lymph node station.

\section{Conclusions and future directions}

Due to the lack of powerful evidence regarding which of the current combined modality therapy is more beneficial on the GC patients after surgery, the standards of the postoperative treatments still differ around the world. To date, the role of adjuvant CT has been established and postoperative $\mathrm{CT}$ is routinely used for D2-dissected GC, however, the value of adding postoperative RT to CT is largely unknown. Several previous randomized studies and meta-analyses provided inconsistent results. Differences between tumor epidemiology (tumor stage at diagnosed), treatment policies, treatment outcomes might explain the results. For the best individual treatment, future trials should focus on predictive markers and particular characteristics at the definitive histological examination. Toxicity and quality of life of a selected high-risk category of patients should be assessed precisely to individualise adjuvant therapy and optimise therapeutic ratio. Though $\geq 85 \%$ of the cohort in SEER database treated after INT-0116 would be eligible for adjuvant RT because they had Stage Ib-IVM0 disease, however, only $30.4 \%$ received adjuvant RT [32]. Knowledge of recurrence patterns (locoregional, peritoneal, or distant/systemic) after curative resection could potentially guide the planning of adjuvant treatment and minimize radiation-related toxic effects. However, until now, only a limited number of retrospective studies had defined the specific location of locoregional recurrence for postoperative RT in GC. To overcome the restricted nature of a retrospective study and provide more solid conclusion of radiation field determination, additional large prospective studies are required to evaluate the optimal individualized target volume construction. In brief, the benefits of adjuvant RT and optimal target volume in GC remain unclear, and are open for future investigations about reliable patient and tumor-related criteria for appropriate patient selection and tailored clear and detailed delineation of the irradiation of lymph node stations.

\section{Abbreviations}

AUC: area under the curve; CRT: chemoradiotherapy; CT: chemotherapy; DFS: disease-free survival; CTV: clinical target volume; 2-D: two-dimensional; 3D-CRT: three-dimensional conformal radiation therapy; GC: gastric cancer; LRRFS: locoregional recurrence-free survival; PTV: planning tumor volume; RT: radiotherapy; IMRT: intensity modulated radiation therapy; FP: 5-fluorouracil/cisplatin; ARTIST: Adjuvant chemoRadiation Therapy in Stomach cancer; STG: subtotal gastrectomy.

\section{Acknowledgements}

The authors appreciate the support from Zhejiang Province Gastric Cancer Diagnosis and Treatment Technology Research Center (no. JBZX-201802).

\section{Competing Interests}

The authors have declared that no competing interest exists.

\section{References}

1. Siegel R, Ma J, Zou Z, Jemal A. Cancer statistics, 2014. CA Cancer J Clin. 2014; 64: 9-29.

2. Bockbrader M, Kim E. Role of intensity-modulated radiation therapy in gastrointestinal cancer. Expert review of anticancer therapy. 2009; 9: 637-47

3. Bonenkamp JJ, Songun I, Hermans J, et al. Randomised comparison of morbidity after D1 and D2 dissection for gastric cancer in 996 Dutch patients. Lancet. 1995; 345: 745-8.

4. Bonenkamp JJ, Hermans J, Sasako M, et al. Extended lymph-node dissection for gastric cancer. The New England journal of medicine. 1999; 340: 908-14.

5. Cuschieri A, Fayers P, Fielding J, et al. Postoperative morbidity and mortality after D1 and D2 resections for gastric cancer: preliminary results of the MRC randomised controlled surgical trial. The Surgical Cooperative Group. Lancet. 1996; 347: 995-9.

6. Songun I, Putter H, Kranenbarg EM, Sasako M, van de Velde CJ. Surgical treatment of gastric cancer: 15-year follow-up results of the randomised nationwide Dutch D1D2 trial. The Lancet Oncology. 2010; 11: 439-49.

7. Tsujinaka T, Fujitani K, Hirao M, Kurokawa Y. Current status of chemoradiotherapy for gastric cancer in Japan. International journal of clinical oncology. 2008; 13: 117-20.

8. Okines A, Verheij M, Allum W, Cunningham D, Cervantes A. Gastric cancer: ESMO Clinical Practice Guidelines for diagnosis, treatment and follow-up. Annals of oncology : official journal of the European Society for Medical Oncology. 2010; 21 Suppl 5: v50-4.

9. D'Angelica M, Gonen M, Brennan MF, Turnbull AD, Bains M, Karpeh MS. Patterns of initial recurrence in completely resected gastric adenocarcinoma. Annals of surgery. 2004; 240: 808-16.

10. Yoo CH, Noh SH, Shin DW, Choi SH, Min JS. Recurrence following curative resection for gastric carcinoma. Br J Surg. 2000; 87: 236-42.

11. Quero L, Guillerm S, Hennequin C. Neoadjuvant or adjuvant therapy for gastric cancer. World journal of gastrointestinal oncology. 2015; 7: 102-10. 
12. Agolli L, Maurizi Enrici R, Osti MF. Adjuvant radiochemotherapy for gastric cancer: Should we use prognostic factors to select patients? World J Gastroenterol. 2016; 22: 1131-8.

13. Macdonald JS, Smalley SR, Benedetti J, Hundahl SA, Estes NC, Stemmermann GN, et al. Chemoradiotherapy after surgery compared with surgery alone for adenocarcinoma of the stomach or gastroesophageal junction. N Engl J Med. 2001; 345: 725-30.

14. Smalley SR, Benedetti JK, Haller DG, et al. Updated analysis of SWOG-directed intergroup study 0116: a phase III trial of adjuvant radiochemotherapy versus observation after curative gastric cancer resection. Journal of clinical oncology : official journal of the American Society of Clinical Oncology. 2012; 30: 2327-33.

15. Norero E, Bustos M, Herrera ME, et al. Postoperative adjuvant treatment for gastric cancer improves long-term survival after curative resection and D2 lymphadenectomy. Results from a Latin American Center. European journal of surgical oncology : the journal of the European Society of Surgical Oncology and the British Association of Surgical Oncology. 2016; 42: 94-102.

16. Kim S, Lim DH, Lee J, et al. An observational study suggesting clinical benefit for adjuvant postoperative chemoradiation in a population of over 500 cases after gastric resection with D2 nodal dissection for adenocarcinoma of the stomach. International journal of radiation oncology, biology, physics. 2005; 63: 1279-85.

17. Park SH, Sohn TS, Lee J, et al. Phase III Trial to Compare Adjuvant Chemotherapy With Capecitabine and Cisplatin Versus Concurrent Chemoradiotherapy in Gastric Cancer: Final Report of the Adjuvant Chemoradiotherapy in Stomach Tumors Trial, Including Survival and Subset Analyses. Journal of clinical oncology : official journal of the American Society of Clinical Oncology. 2015; 33: 3130-6

18. Lee J, Lim DH, Kim S, et al. Phase III trial comparing capecitabine plus cisplatin versus capecitabine plus cisplatin with concurrent capecitabine radiotherapy in completely resected gastric cancer with D2 lymph node dissection: the ARTIST trial. Journal of clinical oncology : official journal of the American Society of Clinical Oncology. 2012; 30: 268-73.

19. Yu JI, Lim DH, Lee J, et al. Necessity of adjuvant concurrent chemo-radiotherapy in D2-resected LN-positive gastric cancer. Radiother Oncol. 2018; [Epub ahead of print].

20. Zhu WG, Xua DF, Pu J, et al. A randomized, controlled, multicenter study comparing intensity-modulated radiotherapy plus concurrent chemotherapy with chemotherapy alone in gastric cancer patients with D2 resection. Radiotherapy and oncology : journal of the European Society for Therapeutic Radiology and Oncology. 2012; 104: 361-6.

21. Kim TH, Park SR, Ryu KW, et al. Phase 3 trial of postoperative chemotherapy alone versus chemoradiation therapy in stage III-IV gastric cancer treated with R0 gastrectomy and D2 lymph node dissection. International journal of radiation oncology, biology, physics. 2012; 84: e585-92.

22. Yu C, Yu R, Zhu W, Song Y, Li T. Intensity-modulated radiotherapy combined with chemotherapy for the treatment of gastric cancer patients after standard D1/D2 surgery. Journal of cancer research and clinical oncology. 2012; 138: 255-9.

23. Huang YY, Yang Q, Zhou SW, et al. Postoperative chemoradiotherapy versus postoperative chemotherapy for completely resected gastric cancer with D2 Lymphadenectomy: a meta-analysis. PloS one. 2013; 8: e68939.

24. Min C, Bangalore S, Jhawar S, et al. Chemoradiation therapy versus chemotherapy alone for gastric cancer after R0 surgical resection: a meta-analysis of randomized trials. Oncology. 2014; 86: 79-85.

25. Zhou ML, Kang M, Li GC, Guo XM, Zhang Z. Postoperative chemoradiotherapy versus chemotherapy for $\mathrm{R} 0$ resected gastric cancer with D2 lymph node dissection: an up-to-date meta-analysis. World journal of surgical oncology. 2016; 14: 209.

26. Kwon $\mathrm{HC}$, Kim MC, $\mathrm{Kim} \mathrm{KH}$, et al. Adjuvant chemoradiation versus chemotherapy in completely resected advanced gastric cancer with D2 nodal dissection. Asia-Pacific journal of clinical oncology. 2010; 6: 278-85.

27. Bamias A, Karina M, Papakostas P, et al. A randomized phase III study of adjuvant platinum/docetaxel chemotherapy with or without radiation therapy in patients with gastric cancer. Cancer chemotherapy and pharmacology. 2010; 65: 1009-21.

28. Kilic L, Ordu C, Ekenel M, et al. Comparison of two different adjuvant treatment modalities for $\mathrm{pN} 3$ gastric cancer patients after D2 lymph node dissection: can we avoid radiotherapy in a subgroup of patients? Med Oncol. 2013; 30: 660.

29. Dikken JL, van Sandick JW, Maurits Swellengrebel HA, et al. Neo-adjuvant chemotherapy followed by surgery and chemotherapy or by surgery and chemoradiotherapy for patients with resectable gastric cancer (CRITICS). BMC cancer. 2011; 11: 329.

30. Cats A, Jansen EPM, van Grieken NCT, et al. Chemotherapy versus chemoradiotherapy after surgery and preoperative chemotherapy for resectable gastric cancer (CRITICS): an international, open-label, randomised phase 3 trial. Lancet Oncol. 2018; 19: 616-28.

31. Wu Q, Li G, Xu F. Resected gastric cancer with D2 dissection: advances in adjuvant chemoradiotherapy and radiotherapy techniques. Expert review of anticancer therapy. 2015; 15: 703-13.

32. Coburn NG, Guller U, Baxter NN, et al. Adjuvant therapy for resected gastric cancer--rapid, yet incomplete adoption following results of intergroup 0116 trial. International journal of radiation oncology, biology, physics. 2008; 70: 1073-80.

33. Liu $\mathrm{D}, \mathrm{Lu} \mathrm{M}, \mathrm{Li} \mathrm{J}$, et al. The patterns and timing of recurrence after curative resection for gastric cancer in China. World J Surg Oncol. 2016; 14: 305 .

34. Li G, Wang J, Hu W, Zhang Z. Radiation-Induced Liver Injury in Three-Dimensional Conformal Radiation Therapy (3D-CRT) for Postoperative or Locoregional Recurrent Gastric Cancer: Risk Factors and Dose Limitations. PloS one. 2015; 10: e0136288.

35. Trip AK, Nijkamp J, van Tinteren H, et al. IMRT limits nephrotoxicity after chemoradiotherapy for gastric cancer. Radiother Oncol. 2014; 112: 289-94.

36. Alani S, Soyfer V, Strauss N, Schifter D, Corn BW. Limited advantages of intensity-modulated radiotherapy over 3D conformal radiation therapy in the adjuvant management of gastric cancer. Int J Radiat Oncol Biol Phys. 2009; 74: 562-6.

37. Trip AK, Sikorska K, van Sandick JW, et al. Radiation-induced dose-dependent changes of the spleen following postoperative chemoradiotherapy for gastric cancer. Radiotherapy and oncology : journal of the European Society for Therapeutic Radiology and Oncology. 2015; 116: 239-44.

38. Wydmanski J, Polanowski P, Tukiendorf A, Maslyk B. Radiation-induced injury of the exocrine pancreas after chemoradiotherapy for gastric cancer. Radiotherapy and oncology : journal of the European Society for Therapeutic Radiology and Oncology. 2016; 118: 535-9.

39. Dennis K, Poon M, Chow E. Nausea and vomiting induced by gastrointestinal radiation therapy: current status and future directions. Current opinion in supportive and palliative care. 2015; 9: 182-8.

40. Schwarz RE, Zagala-Nevarez K. Recurrence patterns after radical gastrectomy for gastric cancer: prognostic factors and implications for postoperative adjuvant therapy. Annals of surgical oncology. 2002; 9: 394-400.

41. Eom BW, Yoon H, Ryu KW, et al. Predictors of timing and patterns of recurrence after curative resection for gastric cancer. Digestive surgery. 2010; 27: 481-6.

42. Smalley SR, Gunderson L, Tepper J, et al. Gastric surgical adjuvant radiotherapy consensus report: rationale and treatment implementation. Int J Radiat Oncol Biol Phys. 2002; 52: 283-93.

43. Nam H, Lim DH, Kim S, et al. A new suggestion for the radiation target volume after a subtotal gastrectomy in patients with stomach cancer. Int J Radiat Oncol Biol Phys. 2008; 71: 448-55.

44. Yoon HI, Chang JS, Lim JS, et al. Defining the target volume for post-operative radiotherapy after D2 dissection in gastric cancer by CT-based vessel-guided delineation. Radiother Oncol. 2013; 108: 72-7.

45. Chang JS, Kim KH, Yoon HI, et al. Locoregional relapse after gastrectomy with D2 lymphadenectomy for gastric cancer. Br J Surg. 2017; 104: 877-84.

46. Lim DH, Kim DY, Kang MK, et al. Patterns of failure in gastric carcinoma after D2 gastrectomy and chemoradiotherapy: a radiation oncologist's view. Br J Cancer. 2004; 91: 11-7.

47. Chang JS, Lim JS, Noh $\mathrm{SH}$, et al. Patterns of regional recurrence after curative D2 resection for stage III (N3) gastric cancer: implications for postoperative radiotherapy. Radiotherapy and oncology : journal of the European Society for Therapeutic Radiology and Oncology. 2012; 104: 367-73.

48. Yu JI, Lim do $\mathrm{H}$, Ahn YC, et al. Effects of adjuvant radiotherapy on completely resected gastric cancer: A radiation oncologist's view of the ARTIST randomized phase III trial. Radiotherapy and oncology : journal of the European Society for Therapeutic Radiology and Oncology. 2015; 117: $171-7$.

49. Maruyama K, Gunven P, Okabayashi K, Sasako M, Kinoshita T. Lymph node metastases of gastric cancer. General pattern in 1931 patients. Ann Surg. 1989; 210: 596-602.

50. Yi Y, Yu J, Li B, et al. Pattern of lymph node metastases and its implication in radiotherapeutic clinical target volume delineation of regional lymph node in patients with gastric carcinoma. Radiother Oncol. 2010; 96: 223-30.

51. Dong W, Li B, Wang J, Song Y, Zhang Z, Fu C. Exploration of the Radiotherapeutic Clinical Target Volume Delineation for Gastric Cancer from Lymph Node Metastases. Anticancer Res. 2017; 37: 4629-34. 
52. Jansen EP, Nijkamp J, Gubanski M, Lind PA, Verheij M. Interobserver variation of clinical target volume delineation in gastric cancer. International journal of radiation oncology, biology, physics. 2010; 77: 1166-70.

53. Moretones C, Leon D, Navarro A, et al. Interobserver variability in target volume delineation in postoperative radiochemotherapy for gastric cancer. A pilot prospective study. Clinical \& translational oncology : official publication of the Federation of Spanish Oncology Societies and of the National Cancer Institute of Mexico. 2012; 14: 132-7.

54. Socha J, Wolakiewicz G, Wasilewska-Tesluk E, et al. Clinical target volume in postoperative radiotherapy for gastric cancer: identification of major difficulties and controversies. Clinical \& translational oncology : official publication of the Federation of Spanish Oncology Societies and of the National Cancer Institute of Mexico. 2016; 18: 480-8. 